\title{
Pós-operatório de paciente oncológico em jejum prolongado: diagnósticos e intervenções de enfermagem
}

\author{
Post-operative oncological patients in prolonged fast: nursing diagnosis and interventions \\ Postoperatorio del paciente oncológico bajo ayuno prolongado: diagnósticos e intervenciones \\ de enfermería
}

Sara Soares Ferreira da Silva' $\bullet$; Rosiane Santos Ferreira' $\bullet$; Ana Cristina Silva Pinto' $\bullet$; Lidiane Bonin de Andrade '๑; Priscilla Manhães Gomes' ๑; Janaína de Medeiros Tavares" ๑

'Universidade Federal do Estado do Rio de Janeiro, Rio de Janeiro, RJ, Brasil;

"Hospital dos Servidores do Estado do Rio de Janeiro, Rio de Janeiro RJ, Brasil

\begin{abstract}
RESUMO
Objetivo: identificar os principais diagnósticos de enfermagem e suas respectivas intervenções em uma população de pacientes oncológicos submetidos a jejum prolongado no pós-operatório. Método: estudo descritivo, documental e transversal. O cenário do estudo foi um hospital federal de ensino no Rio de Janeiro, caracterizado como UNACON. Os dados foram coletados durante 60 dias em prontuários referentes aos anos de 2016 a 2018. Foram analisados 61 prontuários que atendiam aos critérios de inclusão e 208 complicações foram apresentadas pelos pacientes oncológicos. Resultados: Foram definidos oito diagnósticos de enfermagem, entre os quais os mais evidentes foram: Nutrição desequilibrada: menor que as necessidades corporais e Risco de glicemia instável. Onze intervenções de enfermagem foram definidas em consonância com a Taxonomia de NANDA-I e buscaram ser de fácil aplicação na prática assistencial de enfermagem. Conclusão: foi evidenciada uma ampla gama de diagnósticos e intervenções de enfermagem a qual se recomenda sua adoção no processo de enfermagem.

Descritores: Enfermagem Perioperatória; Jejum; Oncologia Cirúrgica; Processo de Enfermagem.
\end{abstract}

\section{ABSTRACT}

Objective: to identify the main nursing diagnoses and respective interventions in a population of cancer patients undergoing prolonged postoperative fasting. Method: descriptive, cross-sectional, documentary study. The study scenario was a federal teaching hospital in Rio de Janeiro, characterized as a high-complexity oncological facility. Data were collected for 60 days from medical records for the years 2016 to 2018. In the 61 medical records that met the inclusion criteria and were analyzed, cancer patients presented 208 complications. Results: eight diagnoses were established, the most in evidence being: nutrition imbalance: less than body requirements; and risk of unstable blood glucose level. Eleven nursing interventions, designed to be easily applied in nursing care practice, were specified in line with the NANDA-I Taxonomy. Conclusion: the wide range of nursing diagnoses and interventions evidenced are recommended for adoption in the nursing process.

Descriptors: Perioperative Nursing; Fasting; Surgical Oncology; Nursing Process.

\section{RESUMEN}

Objetivo: identificar los principales diagnósticos de enfermería y sus respectivas intervenciones en una población de pacientes oncológicos sometidos a ayuno postoperatorio prolongado. Método: estudio descriptivo, transversal, documental. El escenario de estudio fue un hospital universitario federal en Río de Janeiro, caracterizado como una instalación oncológica de alta complejidad. Se recolectaron datos durante 60 días de las historias clínicas de los años 2016 a 2018. En las 61 historias clínicas que cumplieron con los criterios de inclusión y fueron analizadas, los pacientes con cáncer presentaron 208 complicaciones. Resultados: se establecieron ocho diagnósticos, siendo los más evidentes: desequilibrio nutricional: menor que los requerimientos corporales; y riesgo de niveles inestables de glucosa en sangre. Once intervenciones de enfermería, diseñadas para ser fácilmente aplicadas en la práctica del cuidado de enfermería, fueron especificadas de acuerdo con la Taxonomía NANDA-I. Conclusión: la amplia gama de diagnósticos e intervenciones de enfermería evidenciados se recomiendan para su adopción en el proceso de enfermería.

Descriptores: Enfermería Perioperatória; Ayuno; Oncología Quirúrgica; Proceso de Enfermería.

\section{INTRODUÇÃO}

Segundo a OMS, em 2018, 18,1 milhões de pessoas no mundo desenvolveram algum tipo de câncer e destas 9,6 milhões vieram a óbito. A previsão para 2040 é de que esses números praticamente dupliquem ${ }^{1}$. No Brasil, de acordo com o Instituto Nacional de Câncer (INCA), para cada ano do triênio 2020-2022 há previsão de 625 mil novos casos². Para o manejo da doença uma vez já estabelecida, recorre-se à utilização de estratégias como a radioterapia, quimioterapia e/ou cirurgia. De acordo com o INCA, a abordagem cirúrgica enquanto forma tratamento das neoplasias é de suma relevância, podendo promover controle ou até mesmo a cura quando realizada em estágios iniciais, sendo também estratégia de manejo paliativo desta condição. Neste contexto se incluem as cirurgias abdominais ${ }^{3}$. 
No âmbito da abordagem cirúrgica, há o interesse em se considerar a relevância do adequado preparo perioperatório, assim como os preceitos da cirurgia segura, ao qual preza pela redução do risco de dano desnecessário relacionado ao cuidado à saúde a um mínimo aceitável, sendo importante pauta nos períodos pré, intra e pós operatório 4 .

Neste contexto, a nutrição do paciente no perioperatório vem sendo amplamente discutida, uma vez que autores apresentam o paradoxo gerado pelo benefício do jejum, como por exemplo a prevenção da broncoaspiração durante o efeito anestésico e os impactos negativos gerados para recuperação cirúrgica, como o estresse metabólico ${ }^{5,6}$.

Segundo o projeto Aceleração da Recuperação Total Pós-operatória (ACERTO), a abordagem nutricional do paciente cirúrgico deve ser iniciada assim que é promovida sua internação, de forma a zelar pela adequada nutrição em todo processo operatório. É evidenciado também que os pacientes oncológicos estão inclusos como grupo fortemente acometido pela desnutrição hospitalar ${ }^{7}$.

Neste âmbito, corroborado pelo Protocolo ERAS (Enhanced Recovery After Surgery), uma das questões levantadas é a proposta do jejum pós-operatório e seus impactos, sendo afirmado que a dieta oral precoce no pós-operatório está relacionada à diminuição de complicações e aceleração da recuperação. Na Diretriz ACERTO de Intervenções Nutricionais no Perioperatório em Cirurgia Geral Eletiva, é instituído com forte grau de recomendação que a dieta (oral ou enteral) pós-operatória em cirurgias abdominais deve ser iniciada em 24 horas, desde que o paciente não apresente instabilidade hemodinâmica ${ }^{8-11}$

Ressalta-se que, associada a tal discussão, é necessário o preparo do profissional de enfermagem para atuar frente aos possíveis desfechos originados do jejum prolongado, uma vez que tal prática ainda é adotada em diversos serviços de assistência no contexto cirúrgico, apesar das evidências existentes na literatura sobre seus impactos para o paciente ${ }^{11}$.

Ainda, tomando como base ainda a resolução COFEN nำ358/2009 ${ }^{12}$ que dispõe sobre a Sistematização da Assistência em Enfermagem (SAE) e a implementação do processo de enfermagem como prática que deve ser realizada em todos os ambientes que possua a atuação do enfermeiro e, tendo em vista que o diagnóstico, delineamento dos resultados e intervenções são componentes deste processo, se reforça a necessidade de se pensar o pós-operatório por esta óptica. Desta forma, o estudo tem por objetivo identificar os principais diagnósticos de enfermagem e suas respectivas intervenções em uma população de pacientes oncológicos submetidos a jejum prolongado no pósoperatório.

Seu impacto se revela ao contribuir com embasamento científico para profissionais de enfermagem na elaboração do planejamento e implementação de suas atividades, para que assim, obtenham maior êxito e o paciente seja o maior beneficiado.

\section{MÉTODO}

Estudo transversal, descritivo e documental. O cenário de estudo constituiu-se de um hospital de ensino da rede federal do Rio de Janeiro categorizado como Unidade de Assistência de Alta Complexidade em Oncologia (UNACON), o qual realiza procedimentos de média e alta complexidade, tendo em vista a implementação da Sistematização da Assistência de Enfermagem.

A coleta de dados ocorreu do período de janeiro a março do ano de 2019 , sendo analisados os prontuários do setor de cirurgia geral dos anos de 2016 a 2018, por meio de instrumento semiestruturado elaborado pelos autores do presente estudo. Tal instrumento contava com as seguintes questões: data de nascimento, sexo, diagnóstico médico, tipo de cirurgia, período de jejum estipulado para o paciente, tempo real de jejum realizado e complicações apresentadas por tais pacientes no período pós-operatório imediato e mediato, sendo considerado como imediato as primeiras 24 horas a partir da finalização da intervenção cirúrgica e mediato o período de 24 horas após a intervenção cirúrgica até o momento de alta.

Em uma primeira etapa foram avaliados os prontuários, oriundos do setor de cirurgia geral, de pacientes submetidos à cirurgia abdominal nos anos de 2016 a 2018, sendo então elencados 126 prontuários. Na segunda etapa, tal quantitativo foi reavaliado conforme os critérios de inclusão e exclusão, na qual a amostra foi reduzida a 61.

Foram incluídos pacientes submetidos a intervenção cirúrgica no aparelho gastrointestinal como forma de intervenção ao quadro neoplásico e submetidos a jejum prolongado, isto é, maior que 24 horas no período pósoperatório. Foram excluídos os desfechos em óbito. No que se refere às complicações apresentadas pelos pacientes, um total de 208 foram identificadas e utilizadas como base para delineamento dos Diagnósticos de Enfermagem.

Como referenciais teóricos para definição dos Diagnósticos de enfermagem, Resultados e Intervenções de enfermagem utilizou-se a Taxonomia da NANDA Internacional(2018-2020) ${ }^{13}$, Nursing Outcomes Classification NOC(2016) $)^{14}$, Nursing Interventions Classification - NIC(2016 $)^{15}$ e Ligações NANDA-I NOC e NIC Condições Clínicas: Suporte ao Raciocínio Crítico e Assistência de Qualidade $(2012)^{16}$. 
$\mathrm{Na}$ etapa de construção dos diagnósticos de enfermagem foram consideradas as terminologias empregadas nos prontuários dos pacientes pelos profissionais de saúde, assim como as recomendações da taxonomia NANDA-I. Desta forma, foram elencadas as afirmativas de diagnósticos, resultados e intervenções de enfermagem, relacionadas às necessidades dos clientes oncológicos hospitalizados para cuidados de saúde.

Sendo assim, para a composição dos diagnósticos, foram utilizadas as seguintes diretrizes: incluir, obrigatoriamente, um termo do eixo foco e um termo adicional, conforme a necessidade. Para a construção de afirmativas relacionadas a intervenções de enfermagem, foram utilizadas as seguintes diretrizes: incluir, obrigatoriamente, um termo do eixo ação e um termo alvo, além de permitir a inclusão de termos adicionais, conforme a necessidade, dos outros eixos.

Após a construção das afirmativas, essas foram submetidas a um processo de validação de conteúdo por especialistas das áreas de oncologia cirúrgica, enfermagem perioperatória e sistematização da assistência de enfermagem. Por sua vez, foram elaborados dois instrumentos: um para validação das afirmativas de Diagnósticos e Resultados e outro para validação das Intervenções de Enfermagem. Nos referidos instrumentos, foi solicitada a colaboração dos enfermeiros no sentido de apontar a viabilidade de aplicação das afirmativas à área da cirurgia oncológica e na prática profissional. Em caso de discordância quanto às afirmativas, requisitou-se um juiz, para sugestões e/ou adequação.

A pesquisa seguiu as normas da Resolução nํ466/12 ${ }^{17}$, Norma Operacional no001/2013 ${ }^{18}$ e Resolução nำ510/2016 do Conselho Nacional de Saúde e suas complementares ${ }^{19}$. O projeto foi submetido ao Comitê de Ética da instituição proponente e aprovado pelo parecer no3.039.912.

\section{RESULTADOS}

O total de pacientes analisados segundo critérios de inclusão e exclusão foi de 61 , sendo 10 correspondentes ao ano de 2016, 18 ao ano de 2017 e 33 ao ano de 2018. O tempo de jejum, contabilizado em todos os anos analisados ao qual os pacientes foram submetidos, obteve média de $64 \mathrm{~h} 40 \mathrm{~min}(\sigma=2,22)$ e mediana de $42 \mathrm{~h} 16 \mathrm{~min}$, ressalta-se que o tempo máximo de jejum foi de 136h23min (5 dias). Houve mediana de 3 complicações por paciente e média de 3,4 $(\sigma=1,85)$.

A Tabela 1 demonstra as principais complicações apresentadas pelos pacientes estudados, assim como suas distribuições.

\begin{tabular}{|c|c|c|}
\hline Complicação & $\mathbf{n}$ & $f(\%)$ \\
\hline Hipoglicemia & 45 & 21,63 \\
\hline Hipocalemia & 21 & 10,09 \\
\hline Demais complicações com uma ocorrência & 21 & 10,12 \\
\hline Náusea & 19 & 9,13 \\
\hline Êmese & 17 & 8,17 \\
\hline Hipertensão & 11 & 5,28 \\
\hline Dor (geral) & 11 & 5,28 \\
\hline Hiponatremia & 9 & 4,32 \\
\hline Hiperglicemia & 9 & 4,32 \\
\hline Hipotensão & 8 & 3,84 \\
\hline Dor abdominal & 5 & 2,43 \\
\hline Infecção de sítio cirúrgico & 5 & 2,43 \\
\hline Ansiedade & 3 & 1,44 \\
\hline Astenia & 3 & 1,44 \\
\hline Flatulência & 3 & 1,44 \\
\hline Lipotimia & 3 & 1,44 \\
\hline Sudorese & 3 & 1,44 \\
\hline Cefaleia & 2 & 0,96 \\
\hline Alteração do estado mental & 2 & 0,96 \\
\hline Febre & 2 & 0,96 \\
\hline Hipovolemia & 2 & 0,96 \\
\hline Taquicardia & 2 & 0,96 \\
\hline Diarreia & 2 & 0,96 \\
\hline Total & 208 & 100 \\
\hline
\end{tabular}

À análise, observa-se a hipoglicemia como complicação de ocorrência mais frequente, podendo-se sugerir que o tempo de jejum prolongado contribuiu para seu desenvolvimento. 
TABELA 2: Diagnósticos de Enfermagem elencados segundo a Taxonomia da NANDA - Internacional 2018-2020, seus domínios e distribuição de frequências, Rio de Janeiro, RJ, Brasil, 2020.

\begin{tabular}{|c|c|c|c|}
\hline Domínio NANDA-I & Diagnósticos (NANDA-I) & $\mathbf{n}$ & $f(\%)$ \\
\hline 02 - Nutrição & $\begin{array}{l}\text { (00002) Nutrição desequilibrada: menor do que as necessidades corporais } \\
\text { relacionada a ingestão alimentar insuficiente evidenciada por ingestão de alimentos } \\
\text { menor que a ingestão diária recomendada. } \\
\text { (00179) Risco de glicemia instável evidenciado por ingestão alimentar insuficiente. } \\
\text { (00195) Risco de desequilíbrio eletrolítico evidenciado por diarreia e vômito. }\end{array}$ & $\begin{array}{l}61 \\
19\end{array}$ & $\begin{array}{c}100 \% \\
31,15 \%\end{array}$ \\
\hline 03 - Eliminação e troca & $\begin{array}{l}\text { (00197) Risco de motilidade gastrointestinal disfuncional evidenciado por alteração } \\
\text { nos hábitos alimentares, estressores e desnutrição. }\end{array}$ & 61 & $100 \%$ \\
\hline 11 - Segurança e proteção & (00246) Risco de recuperação cirúrgica retardada evidenciado por desnutrição e dor. & 61 & $100 \%$ \\
\hline $\begin{array}{l}09 \text { - Enfrentamento/ tolerância } \\
\text { ao estresse }\end{array}$ & $\begin{array}{l}\text { (00146) Ansiedade relacionado a estressores evidenciado por náusea, diarreia, } \\
\text { hipotensão e dor abdominal. }\end{array}$ & 32 & $52,50 \%$ \\
\hline 04- Atividade/ repouso & $\begin{array}{l}\text { (00032) Padrão respiratório ineficaz relacionado a dor e ansiedade evidenciado por } \\
\text { dispneia }\end{array}$ & 21 & $34,40 \%$ \\
\hline 12 - Conforto & $\begin{array}{l}\text { (00132) Dor aguda relacionada a agente físico lesivo evidenciado por expressão } \\
\text { facial e autorrelato de dor. }\end{array}$ & 17 & $27,90 \%$ \\
\hline
\end{tabular}

A partir das complicações apresentadas foram identificados 8 diagnósticos, dentre eles 3 (37,5\%) pertencentes ao domínio Nutrição e 1 (12,5\%) atribuído a cada um dos demais domínios: Segurança e Proteção, Eliminação e troca, Atividade e repouso, Conforto e Enfrentamento/tolerância ao estresse. Ainda, quanto a distribuição de frequências dos diagnósticos: Risco de desequilíbrio eletrolítico, Padrão respiratório ineficaz e Dor aguda obtiveram as menores frequências.

A Figura 1 apresenta os resultados esperados para cada diagnóstico identificado e suas respectivas intervenções de enfermagem.

\begin{tabular}{|c|c|c|}
\hline Diagnósticos de Enfermagem (NANDA-I) & Resultados de enfermagem esperados (NOC) & Intervenções de enfermagem (NIC) \\
\hline $\begin{array}{l}\text { (00002) Nutrição desequilibrada: menor do que as } \\
\text { necessidades corporais relacionada a ingestão } \\
\text { alimentar insuficiente evidenciada por ingestão de } \\
\text { alimentos menor que a ingestão diária } \\
\text { recomendada. }\end{array}$ & $\begin{array}{l}\text { (1005) Estado nutricional: Indicadores bioquímicos } \\
\text { (1008) Estado Nutricional: Ingestão de alimentos e líquidos }\end{array}$ & (1160) Monitoração nutricional \\
\hline $\begin{array}{l}\text { (00179) Risco de glicemia instável evidenciado por } \\
\text { ingestão alimentar insuficiente. }\end{array}$ & $\begin{array}{l}\text { (1005) Estado nutricional: Indicadores bioquímicos } \\
\text { (1008) Estado Nutricional: Ingestão de alimentos e líquidos }\end{array}$ & $\begin{array}{l}\text { (1160) Monitoração nutricional } \\
\text { (2130) Controle da Hipoglicemia }\end{array}$ \\
\hline $\begin{array}{l}\text { (00132) Dor aguda relacionada a agente físico lesivo } \\
\text { evidenciada por expressão facial e autorrelato de } \\
\text { dor. }\end{array}$ & (1605) Controle da dor & (1400) Controle da dor \\
\hline $\begin{array}{l}\text { (00195) Risco de desequilíbrio eletrolítico } \\
\text { evidenciado por diarreia e vômito. }\end{array}$ & $\begin{array}{l}\text { (2305) Recuperação cirúrgica: pós-operatório imediato } \\
\text { (1015) Função Gastrointestinal }\end{array}$ & $\begin{array}{l}\text { (1450) Controle da Náusea } \\
\text { (1570) Controle do Vômito } \\
\text { (2080) Controle Hidroeletrolítico } \\
\text { (1400) Controle da dor } \\
\text { (0430) Controle Intestinal }\end{array}$ \\
\hline $\begin{array}{l}\text { (00197) Risco de motilidade gastrointestinal } \\
\text { disfuncional evidenciado por alteração nos hábitos } \\
\text { alimentares, estressores e desnutrição. }\end{array}$ & $\begin{array}{l}\text { (2305) Recuperação cirúrgica: pós-operatório imediato } \\
\text { (1008) Estado Nutricional: Ingestão de alimentos e líquidos }\end{array}$ & $\begin{array}{l}\text { (1450) Controle da Náusea } \\
\text { (1570) Controle do Vômito } \\
\text { (2080) Controle Hidroeletrolítico } \\
\text { (1400) Controle da dor } \\
\text { (1160) Monitoração nutricional } \\
\text { (0430) Controle Intestinal }\end{array}$ \\
\hline $\begin{array}{l}\text { (00246) Risco de recuperação cirúrgica retardada } \\
\text { evidenciado por desnutrição e dor. }\end{array}$ & $\begin{array}{l}\text { (2305) Recuperação cirúrgica: pós-operatório imediato } \\
\text { (1008) Estado Nutricional: Ingestão de alimentos e líquidos } \\
\text { (1605) Controle da dor }\end{array}$ & $\begin{array}{l}\text { (1400) Controle da dor } \\
\text { (1160) Monitoração nutricional } \\
\text { (2080) Controle Hidroeletrolítico }\end{array}$ \\
\hline $\begin{array}{l}\text { (00032) Padrão respiratório ineficaz relacionado a } \\
\text { dor e ansiedade evidenciado por dispneia. }\end{array}$ & $\begin{array}{l}\text { (0402) Estado respiratório: Troca gasosa } \\
\text { (0410) Estado respiratório: Permeabilidade de vias aéreas } \\
\text { (1605) Controle da dor }\end{array}$ & $\begin{array}{l}\text { (3350) Monitoração respiratória } \\
\text { (5820) Redução da ansiedade } \\
\text { (1605) Controle da dor }\end{array}$ \\
\hline $\begin{array}{l}\text { (00146) Ansiedade relacionada a estressores } \\
\text { evidenciada por náusea, diarreia, hipotensão e dor } \\
\text { abdominal. }\end{array}$ & $\begin{array}{l}\text { (1211) Nível de Ansiedade } \\
\text { (0004) Sono } \\
\text { (1618) Controle de Náuseas e Vômitos } \\
\text { (2102) Nível de dor } \\
\text { (1605) Controle da dor }\end{array}$ & $\begin{array}{l}\text { (5880) Técnica para acalmar } \\
\text { (5380) Aumento da segurança } \\
\text { (1450) Controle da Náusea } \\
\text { (1570) Controle do Vômito } \\
\text { (1400) Controle da dor }\end{array}$ \\
\hline
\end{tabular}

FIGURA 1: Diagnósticos de enfermagem e respectivos resultados de enfermagem (NOC) e intervenções de enfermagem (NIC) - Rio de Janeiro, RJ, Brasil, 2020. 


\section{DISCUSSÃo}

À análise do grupo amostral, é evidente como fatores fundamentais para o bem-estar físico e psíquico foram prejudicados. Visando encontrar possíveis soluções, no presente estudo são propostos diagnósticos de enfermagem, resultados esperados e suas respectivas intervenções de enfermagem.

A partir dos diagnósticos identificados é possível perceber que metade é categorizada como diagnóstico de risco, demonstrando sua relevância na atuação assistencial. Tal achado é corroborado em demais estudos ${ }^{20-22}$, nos quais tal categoria foi predominante, evidenciando que a atuação do enfermeiro é notável tanto para resolução de problemas já estabelecidos, quanto para sua prevenção.

Sabe-se que a abordagem nutricional é de grande relevância no período perioperatório devido a sua potencialidade de gerar alterações em diversos sistemas orgânicos. Em estudo ${ }^{23}$ realizado sobre diagnósticos de enfermagem do domínio Nutrição em pacientes em pós-operatório, no qual $70 \%$ das cirurgias foram abdominais, concluiu-se que a maioria dos pacientes $(58,8 \%)$ apresentou o diagnóstico Nutrição desequilibrada: menor que as necessidades corporais ${ }^{23}$.

Enfatiza-se, ainda, a relevância da atuação do enfermeiro neste contexto, devido à sua potencialidade de prevenção de tal complicação, contribuição para adequada recuperação pós-operatória e, consequentemente, para a diminuição do tempo de permanência hospitalar do paciente, visto que realiza a identificação oportuna de fragilidades nutricionais do paciente e despende tempo prolongado de contato com o mesmo.

Assim, no diagnóstico Nutrição desequilibrada: menor que as necessidades corporais propõe-se como resultados: Estado Nutricional: Indicadores bioquímicos (1005) e Estado nutricional: Ingestão de alimentos e líquidos(1008). Como intervenção propõe-se a Monitoração nutricional(1160), sendo suas atividades: Pesar o paciente; Monitorar turgor da pele; Identificar anormalidades no cabelo; Monitorar náuseas e vômitos; Identificar anormalidades do intestino; Conduzir testes laboratoriais para monitorar resultados e Monitorar o estado mental ${ }^{13-16}$.

Ainda no âmbito da nutrição, os níveis glicêmicos são marcadores de grande estima para avaliação de pacientes no perioperatório, sendo demonstrado em estudos que o metabolismo da glicose sofre ampla modificação. Ainda que, no presente estudo, a população avaliada manifestou majoritariamente hipoglicemia como complicação pósoperatória, alguns estudos relacionam também o desenvolvimento da hiperglicemia com desencadeamento favorecido pelo jejum prolongado ${ }^{5,11}$. Portanto, no contexto pós-operatório é de grande valia o norteamento através do diagnóstico Risco de glicemia instável, sendo tal fato reforçado em estudo semelhante no qual houve concordância de $80 \%$ entre avaliadores de um material teórico voltado para diagnósticos de enfermagem no período perioperatório ${ }^{20}$.

Desta forma, para contribuir com efetivo planejamento e intervenção nesta desestabilização fisiológica recomenda-se a aplicação do diagnóstico Risco de glicemia instável e suas respectivas intervenções já parcialmente discutidas anteriormente, uma vez que compartilha com demais diagnósticos o resultado Monitorização Nutricional (1160) e suas intervenções. Contudo acrescenta-se as atividades da intervenção Controle da Hipoglicemia (2130), pois conta com medidas de prevenção adequadas ao contexto de um paciente de risco, sendo elas: Identificar o paciente com risco de hipoglicemia; Determinar reconhecimento de sinais e sintomas de hipoglicemia; Monitorar níveis de glicose no sangue e Administrar glicose por via endovenosa, segundo indicado ${ }^{13-16}$.

Outra complicação evidente neste estudo consiste na dor, onde 17 registros de relatos de dor foram contabilizados. Em estudo ${ }^{24}$ conduzido com pacientes em contexto semelhante ao do presente artigo, a presença da dor foi amplamente relacionada ao desenvolvimento de ansiedade e depressão, sendo indicada também como fator predisponente para piora da qualidade do sono ${ }^{24}$. Destaca-se, ainda, que tal sintoma é recorrente em pacientes oncológicos durante toda a sua trajetória com a doença e não somente no perioperatório, sendo de suma importância que o enfermeiro tenha preparo e recursos para atender às necessidades relacionadas à dor visando proporcionar 0 bem estar do cliente e evitar tais repercussões negativas ${ }^{22}$.

Deste modo, para o diagnóstico Dor aguda elencou-se como resultado prioritário o Controle da dor(1605) e sua respectiva intervenção Controle da dor(1400), sendo elencadas as seguintes atividades: Fazer avaliação abrangente da dor para incluir a localização, início/duração, frequência, qualidade, intensidade da dor e fatores precipitantes; Observar sinais não verbais de desconforto; Explorar com o paciente fatores que melhoram/pioram a dor; Avaliar com a equipe de saúde a efetividade das medidas que foram elencadas para alívio da dor; Orientar sobre o uso de técnicas não farmacológicas; Controlar os fatores ambientais que interferem na resposta à dor (como iluminação, ruídos e temperatura); Utilizar medidas de controle da dor antes da dor se tornar severa; Promover sono e repouso adequado ao paciente ${ }^{13-16}$.

Tais intervenções são corroboradas por revisão integrativa ${ }^{25}$, que demonstrou convergência dos estudos para o fato de que para gestão da dor é indicado avaliar, intervir e reavaliar após cada intervenção implementada, sendo 
indicado observar fatores como diâmetro pupilar, movimentação, alterações respiratórias, expressão facial e inquietude, sendo indicado também o uso de escalas validadas para tal propósito.

No que se refere às alterações hidroeletrolíticas, é conhecida sua forte correlação com êmese e quadros diarreicos. No presente artigo, 32 pacientes (15,38\%) apresentaram desequilíbrios eletrolíticos, entre eles de potássio, magnésio e sódio, e 19 (9,1\%) apresentaram êmese e quadros diarreicos. Em estudo de revisão sobre complicações pós-operatórias ${ }^{26}$, a ocorrência de êmese foi relatada em oito artigos (23,3\%), sendo apontado ainda que, juntamente com a náusea, tal complicação foi a principal na categoria de complicações do trato gastrointestinal. Tal estudo também demonstrou que, dentre demais fatores, há relação destas complicações com o tratamento prévio utilizando quimioterápicos e radioterápicos, contexto esse ao qual a população do presente artigo está inserida por se tratar de pacientes oncológicos.

Consequentemente, sugere-se o diagnóstico Risco de desequilíbrio eletrolítico para melhor manejo desta situação, contando como um de seus resultados esperados Função gastrointestinal(1015) e como intervenção proposta o Controle Intestinal(0430). Ainda, outro resultado selecionado consiste no Recuperação cirúrgica: pós-operatório imediato(2135) sendo elencadas como intervenções possíveis: Controle da dor(1605), já esmiuçada em diagnósticos anteriores; Controle da Náusea(1450); Controle do Vômito(1570) e Controle hidroeletrolítico(2080) ${ }^{13-16}$.

Para o resultado Controle intestinal propõe-se as atividades: Observar data da última evacuação; Avaliar consistência, formato, volume e cor; Monitorar ruídos intestinais; Relatar aumento ou diminuição na frequência dos ruídos intestinais e/ou ruídos intestinais com som elevado e Monitorar ocorrência de sinais e sintomas de diarreia, constipação e impactação $0^{14-16}$.

Em Controle da Náusea, as atividades elencadas foram: Realizar avaliação completa das náuseas incluindo tempo de duração, intensidade, frequência e fatores precipitantes; Controlar os fatores ambientais que podem provocar náuseas; Promover adequado repouso e sono para facilitar o alívio das náuseas e Certificar-se da efetividade de medicamentos antieméticos que são prescritos ${ }^{14-16}$.

As atividades propostas para Controle do Vômito são: Avaliar êmese em relação a cor, consistência, presença de sangue, horário e força utilizada; Mensurar ou estimar o volume da êmese; Sugerir uso de saco plástico para o paciente vomitar; Controlar fatores ambientais que possam provocar vômitos; Posicionar o paciente para prevenir aspiração; Fornecer apoio físico durante vômito; Realizar higiene oral e do nariz; Monitorar o equilíbrio hidroeletrolítico e Encorajar o repouso ${ }^{14-16}$.

Para Controle hidroeletrolítico evidencia-se: Monitorar quanto a níveis séricos anormais eletrolíticos, conforme disponibilidade; Monitorar quanto a alterações pulmonares ou cardíacas indicativas de excesso de líquidos ou desidratação; Monitorar manifestações de desequilíbrio eletrolítico; Observar membranas bucais do paciente, esclera e a pele quanto a indicações de alteração de equilíbrio hídrico ou eletrolítico (p. ex., ressecamento, cianose e icterícia); Administrar eletrólitos suplementares prescritos, conforme apropriado; Administrar resinas de ligação excretoras de eletrólitos, conforme apropriado.

Ainda, diante do quadro de jejum prolongado, se apresentam diversas alterações da peristalse, sendo relevante o diagnóstico Risco de motilidade gastrointestinal disfuncional. Para tal, adotou-se os resultados Recuperação cirúrgica: pós-operatório imediato(2305) e Estado Nutricional: Ingestão de alimentos e líquidos(1008), sendo estes já elucidados nos diagnósticos anteriores ${ }^{13-16}$

Diante dos diversos fatores citados é necessária a atenção do profissional de enfermagem à vulnerabilidade de tais pacientes quanto a efetiva recuperação cirúrgica. No presente artigo, 61 (100\%) pacientes foram contemplados com o diagnóstico Risco de recuperação cirúrgica retardada, sendo principalmente relacionado à condição de jejum prolongado ao qual todos foram submetidos com consequente prejuízo ao estado nutricional ${ }^{27}$. Tal conduta é apoiada por estudo ${ }^{21}$, conduzido com pacientes em período transoperatório, majoritariamente de cirurgia geral $(40 \%, n=26)$, sendo tal diagnóstico delimitado para $23 \%(n=15)$ dos pacientes do grupo amostral.

Assim, para o diagnóstico Risco de recuperação cirúrgica retardada elencou-se como resultados esperados: Recuperação cirúrgica: pós-operatório imediato(2305); Estado Nutricional: Ingestão de alimentos e líquidos(1008) e Controle da dor(1605), sendo suas respectivas intervenções já expostas anteriormente ${ }^{13-16}$.

No que tange ao sistema respiratório, em estudo realizado visando construir um material teórico com diagnósticos, resultados e intervenções de enfermagem para o período transoperatório ${ }^{20}$, o diagnóstico Padrão respiratório ineficaz se apresentou como elemento de composição do material, com concordância superior a 80\% entre os especialistas que avaliaram o mesmo. Ainda, em estudo de revisão sobre complicações no pós-operatório ${ }^{26}$, as complicações relacionadas ao sistema respiratório foram apresentadas em 13 artigos (43,3\%), sendo apontados como principais manifestações dos pacientes: incapacidade de respirar profundamente; hipoxemia leve a moderada; dispneia; hipoxemia grave e dessaturação, demonstrando, portanto, grande importância neste contexto. 
Com isso, o diagnóstico Padrão respiratório ineficaz conta com os seguintes resultados esperados: Estado respiratório: Troca gasosa(0402); Estado respiratório: Permeabilidade de vias aéreas(0410) e Controle da dor(1605) ${ }^{13-16}$.

Como intervenções voltadas para os resultados Estado respiratório: Troca gasosa e Estado respiratório: Permeabilidade de vias aéreas, elencou-se como intervenções de enfermagem: Monitoração respiratória(3350); Redução da ansiedade(5820) e Controle da dor(1400). As atividades são elencadas a seguir, evidenciando-se que o resultado Controle da dor e suas intervenções se encontram descritos em parágrafos anteriores ${ }^{13-16}$.

Em Monitoração respiratória são destacadas as seguintes atividades: Monitorar frequência, ritmo, profundidade e esforço das respirações; Observar os movimentos torácicos, notando a simetria, uso dos músculos acessórios e retração da musculatura supraclavicular e intercostal; Monitorar quanto a respirações ruidosas, como estridores e roncos; Monitorar padrões respiratórios e Determinar a necessidade de aspiração após a ausculta de estertores ou roncos sobre as grandes vias aéreas ${ }^{14-16}$.

Em Redução da ansiedade são atividades possíveis: Utilizar abordagem clara e tranquilizadora; Explicar os procedimentos, inclusive sensações que provavelmente serão vivenciadas durante o procedimento; Permanecer com o paciente para promover segurança e diminuir o medo; Encorajar a família a permanecer com o paciente, conforme indicado; Fornecer objetos que simbolizem segurança; Massagear as costas/pescoço, conforme apropriado, Encorajar a verbalização dos sentimentos, das percepções e dos medos; Identificar mudanças no nível de ansiedade; Proporcionar atividades de diversão voltadas à redução da tensão e Auxiliar o paciente a identificar situações que precipitam a ansiedade ${ }^{14-16}$.

Em estudo ${ }^{24}$, conduzido com pacientes em pós-operatório mediato de cirurgias oncológicas, ao abordar os temas ansiedade e depressão, foi relatado que a maioria dos pacientes apresentaram ansiedade leve a moderada no pósoperatório (40 a 50\%) e esta foi apresentada como um dos fatores contribuintes piora da qualidade de sono. Portanto, entende-se a importância de o enfermeiro atuar neste fator em todo perioperatório. Ainda, em estudo ${ }^{20}$ voltado para construção de material teórico de diagnósticos e intervenções de enfermagem para o período transoperatório, o diagnóstico Ansiedade foi elencado como importante constituinte do material, obtendo concordância de mais de $80 \%$ dos experts avaliadores.

Desta forma, em Ansiedade propõe-se como resultados esperados Nível de Ansiedade(1211); Controle de Náuseas e Vômitos(1618); Sono(0004); Nível de dor(2102) e Controle da dor(1605). Sendo suas respectivas intervenções: Técnica para acalmar(5880); Aumento da segurança(5380); Controle da Náusea(1450); Controle do Vômito(1570) e Controle da $\operatorname{dor}(1400)^{13-16}$.

$\mathrm{Na}$ intervenção Técnica para acalmar elencou-se as seguintes atividades: Manter contato visual com paciente; Identificar pessoas significativas cuja presença possa ajudar o paciente; Massagear fronte do paciente, conforme apropriado; Orientar o paciente quanto aos métodos para diminuir a ansiedade (p. ex., técnicas de respiração lenta, distração, visualização, meditação, relaxamento muscular progressivo, ouvir música suave), como apropriado. Acrescenta-se ainda como estratégia elucidada em estudos, a utilização do apoio social e espiritual visto seus benefícios como diminuição de desesperança, ansiedade, melhora do quadro de saúde e bem-estar espiritual ${ }^{15,16,28-30}$.

As intervenções voltadas para o Aumento de segurança em muito se assemelham às recomendadas como Técnica para acalmar, sendo acrescidas as atividades: Ficar próximo ao paciente e garantir proteção e segurança durante os períodos de ansiedade; Apresentar mudanças de forma gradual; Discutir as próximas mudanças (p. ex., transferência de enfermaria) antes do evento em si e Evitar causar situações emocionais intensas ${ }^{14-16}$.

\section{CONCLUSÕES}

O estudo evidenciou as vulnerabilidades de um grupo de pacientes oncológicos submetidos ao jejum prolongado no pós-operatório buscando demonstrar que as ações da enfermagem, tanto para resolução de um problema já estabelecido assim como para sua prevenção, são de total relevância, podendo ser determinantes para recuperação deste grupo de pacientes.

Assim, com base neste contexto específico, uma ampla gama de diagnósticos e intervenções de enfermagem foi identificada e recomendada para aplicação na prática clínica durante o processo de enfermagem nas etapas de diagnósticos, planejamento da assistência e avaliação de enfermagem. Com vistas a facilitar e promover a assistência de enfermagem com maior especificidade e qualidade a este grupo de pacientes, além de contribuir com novos conhecimentos sobre o cuidado aos pacientes oncológicos.

Como limitações, o estudo não contou com utilização de testes de hipótese para desenvolvimento dos diagnósticos propostos, sendo realizados através de inferência com concordância entre os pesquisadores. Ademais, a quantidade amostral pode não ser suficiente para fiabilidade dos dados, portanto, recomenda-se a elaboração de estudos com maior número amostral com o propósito de extrapolar os achados do presente artigo. 


\section{REFERÊNCIAS}

1. World Health Organization. WHO report on cancer: setting priorities, investing wisely and providing care for all [Internet]. Geneva: WHO; 2020. [cited 2020 Feb 04]; Available from: https://apps.who.int/iris/handle/10665/330745;jsessionid=2722B5EBF3812CFF2E489833DF9EE9A8

2. Instituto Nacional de Câncer José Alencar Gomes da Silva. Estimativa 2020 : incidência de câncer no Brasil [Internet]. Rio de Janeiro (RJ): INCA; 2019 [cited 2020 Feb 04]; Available from: https://www.inca.gov.br/sites/ufu.sti.inca.local/files/media/document/estimativa-2020-incidencia-de-cancer-no-brasil.pdf

3. INCA. Instituto Nacional de Câncer [site de Internet]. Tratamento do Câncer Cirurgia. [cited 2020 Feb 04]; Available from: https://www.inca.gov.br/tratamento/cirurgia

4. Ribeiro WA, Mattos I de F, Morais MC de, Souza DM da S, Couta CS, Martins LM. Safe Surgery: Nursing Leading the Patient Safety at the Surgical Center. Rev. Pró-UniverSUS [Internet]. 2019 [cited 2020 abr 18]; 10(1):66-71. Available from: http://editora.universidadedevassouras.edu.br/index.php/RPU/article/view/1684

5. Campos SBG, Barros-Neto JA, Guedes G da S, Moura FA. Pre-operative fasting: Why abbreviate? ABCD Arq. Bras. Cir. Dig. [Internet]. 2018 [cited 2020 Apr 18]; 31(2): e1377. Available from: http://www.scielo.br/scielo.php?script=sci_arttext\&pid=S0102-67202018000200508

6. Tudor-Drobjewski BA, Marhofer P, Kimberger O, Huber WD, Roth G, Triffterer L. Randomised controlled trial comparing preoperative carbohydrate loading with standard fasting in paediatric anaesthesia. Br. J. Anaesth [Internet]. 2018 [cited 2020 Apr 18]; 121(3):656-61. Available from: https://bjanaesthesia.org/article/S0007-0912(18)30381-7/fulltext

7. Periop. Medicina Perioperatória [site de internet]. Projeto Acerto. [cited 2020 Apr 18]. Available from: https://www.periop.com.br/copia-jejum-pre-operatorio

8. de-Aguilar-Nascimento JE, Salomão AB, Waitzberg DL, Dock-Nascimento DB, Correa MITD, Campos ACL et al. ACERTO guidelines of perioperative nutritional interventions period in elective general surgery. Rev. Col. Bras. Cir. [Internet]. 2017 [cited $2020 \mathrm{Apr}$ 18]; 44(6):633-48. Available from: http://www.scielo.br/scielo.php?pid=S010069912017000600633\&script=sci_abstract\&tlng=pt

9. Ota H, Ikenaga M, Hasegawa J, Murata K, Miyake Y, Mizushima T, et al. Safety and efficacy of an "enhanced recovery after surgery" protocol for patients undergoing colon cancer surgery: a multi-institutional controlled study. Surg. Today [Internet]. 2017 [cited 2020 Apr 18]; 47(6):668-75. Available from: https://www.ncbi.nlm.nih.gov/pubmed/27688031

10. Teixeira UF, Fontes PRO, Conceição CWN, Farias CAT, Fernandes D, Ewald IP, et al. Implementation of enhanced recovery after colorectal surgery (ERAS) protocol: Initial results of the first Brazilian experience. ABCD Arq. Bras. Cir. Dig. [Internet]. 2019 [cited 2020 Apr 18]; 32(1). Available from: http://www.scielo.br/scielo.php?script=sci_arttext\&pid=S0102-67202019000100306

11. Pereira NC, Turrini RNT, Poveda VB. Perioperative fasting time among cancer patients submitted to gastrointestinal surgeries. Rev. Esc. Enferm. USP [Internet]. 2017 [cited 2020 Apr 18];51. Available from: http://www.scielo.br/scielo.php?script=sci_arttext\&pid=S0080-62342017000100425

12. Conselho Federal de Enfermagem (Brasil). Resolução nำ358, de 15 de outubro de 2009. Dispõe sobre a Sistematização da Assistência de Enfermagem e a implementação do Processo de Enfermagem em ambientes, públicos ou privados, em que ocorre o cuidado profissional de Enfermagem, e dá outras providências. Diário Oficial da União 23 out 2009; Seção 1.

13. NANDA International. Diagnósticos de enfermagem da NANDA-I: definições e classificação 2018-2020. 11a ed. Tradução de Regina Machado Garcez. Porto Alegre: Artmed; 2017.

14. Moorhead S, Johnson M, Maas ML, Swanson E. Classificação dos resultados de enfermagem (NOC). 5a ed. Rio de Janeiro: Elsevier; 2016.

15. Bulechek GM, Butcher HK, Dochterman JM, Wagner CM. Classificação das intervenções de enfermagem (NIC). 6ª ed. Rio de Janeiro: Elsevier; 2016.

16. Johnson M, Moorhead S, Bulechek G, Butcher H, Maas M, Swanson E. Ligações NANDA NOC-NIC Condições clínicas suporte ao raciocínio e assistência de qualidade. 3a ed. Rio de Janeiro: Elsevier; 2013.

17. Ministério da Saúde (Br). Resolução no466, de 12 de dezembro de 2012. Estabelece diretrizes e normas regulamentadoras de pesquisas envolvendo seres humanos. Diário Oficial da União 13 jun 2013; Seção1.

18. Ministério da Saúde (Br). Norma Operacional n0001, de 11 e 12 de setembro de 2013. Dispõe sobre a organização e funcionamento do Sistema CEP/CONEP, e sobre os procedimentos para submissão, avaliação e acompanhamento da pesquisa e de desenvolvimento envolvendo seres humanos no Brasil. [cited 2020 Apr 18]; Available from: http://www.hgb.rj.saude.gov.br/ceap/Norma_Operacional_001-2013.pdf.

19. Ministério da Saúde (Br). Resolução n510, de 07 de abril de 2016. Dispõe sobre a organização e funcionamento do Sistema CEP/CONEP, e sobre os procedimentos para submissão, avaliação e acompanhamento da pesquisa e de desenvolvimento envolvendo seres humanos no Brasil. Diário Oficial da União 24 mai 2016; Seção1.

20. Berwanger DC, Matos FG de OA, Alves DA, Oliveira JLC de. Links between diagnostics, results and nursing interventions for patients in the transoperative period. Rev. SOBECC [Internet]. 2018 [cited 2020 Apr 18]; 51; 23(4):195-204. Available from: https://revista.sobecc.org.br/sobecc/article/view/418/pdf

21. Melo UG, Santana RF, Do Carmo TG, Lopes MVDO. Nursing diagnoses in the perioperative period: cross mapping. Rev. SOBECC [Internet]. 2019 [cited 2020 Apr 18]; 24(4):193-9. Available from: https://revista.sobecc.org.br/sobecc/article/view/521/pdf

22. da Silva MR, Silva D de O, dos Santos TI, de Oliveira PP, Rodrigues AB, Barbosa DA. Mapping of nursing diagnoses, results and interventions in an oncology unit. Rev. enferm. UERJ [Internet]. 2017 [cited 2020 Apr 18]; 25(1). Available from: https://www.epublicacoes.uerj.br/index.php/enfermagemuerj/article/view/15133/23843 
23. Araújo JNM, Moura LA, Araújo MG, Pitombeira DO, Fernandes APNL, Allyne FV. Nutritional clinical indicators of NANDA international in post operatory patients. Rev. Enferm. UFSM [Internet]. 2016 [cited 2020 Apr 18]; 6(4): 559-68. Available from: https://periodicos.ufsm.br/reufsm/article/view/21472/pdf

24. Barbosa LK, Cordeiro Silva JS, Monteiro de Almeida AC, Almeida Leroy PL, Brom Vieira ME. Anxiety, depression and sleep quality in mediate postoperative oncologic surgery. Rev Bras Pesq. Saúde [Internet]. 2019 [cited 2020 Apr 18]; 20(4):71-82. Available from: https://periodicos.ufes.br/rbps/article/view/24600

25. Peiter C, Caminha M, Lanzoni G, Erdmann A. Managing nursing care delivery to cancer patients in a general hospital: a Grounded Theory. Rev. Enf. Ref [Internet]. 2016 [cited 2020 Apr 18] ; IV Série(11): 61-9. Available from: http://www.scielo.mec.pt/pdf/ref/vserlVn11/serlVn11a07.pdf

26. Campos MPDA, Dantas DV, Silva LSL, Santana JFNB, Oliveira DC, Fontes LL. Complications in the post-anesthesia care unit: an integrative review. Rev SOBECC [Internet]. 2018 [cited 2020 Apr 18]; 23(3):160-8. Available from: https://revista.sobecc.org.br/sobecc/article/view/385

27. Bazzi NB, Leal V, Lira Júnior HF, Santos JM, Ferreira MG, Zeni LAZR. Nutritional status and fast time in elective colorectal surgery. Nutr. clín. diet. hosp. [Internet]. 2016 [cited 2020 Apr 18]; 36(2):103-10. Available from: https://pesquisa.bvsalud.org/portal/resource/pt/ibc-153512

28. Xing L, Guo X, Bai L, Qian J, Chen J. Are spiritual interventions beneficial to patients with cancer?: A meta-analysis of randomized controlled trials following PRISMA. Medicine (Baltimore) [Internet]. 2018 [cited 2020 Apr 18];97(35):e11948. Available from: https://www.ncbi.nlm.nih.gov/pubmed/30170390

29. Silva L, Poiares I, Machado C, Lenhani B, Guimarães P, Kalinke L. Religion/spirituality and social support in improving the quality of life of patients with advanced cancer. Rev. Enf. Ref. [Internet]. 2019 [cited 2020 Apr 18]; IV Série (23):111-20. Available from: http://www.scielo.mec.pt/scielo.php?script=sci_arttext\&pid=S0874-02832019000400012

30. Jaramillo RG, Monteiro PS, Borges S. Religious/spiritual coping: a study with family caregivers of children and adolescents undergoing chemotherapy. Cogitare Enferm. [Internet]. 2019 [cited 2020 Apr 18]; 24(0). Available from:

https://revistas.ufpr.br/cogitare/article/view/62297 